\title{
Two Cases of Scimitar Syndrome: An Adolescent and an Older Adult with Late Diagnosis
}

\author{
Gholamreza Omrani ${ }^{1}$, Maziar Gholampour Dehaki ${ }^{1}$, Reza Abbaszadeh ${ }^{1}$, Ali Sadeghpour Tabaee ${ }^{1}$ and \\ Hassan Tatari ${ }^{1, *}$ \\ ${ }^{1}$ Rajaie Cardiovascular Medical and Research Center, Iran University of Medical Sciences, Tehran, Iran \\ "Corresponding author: Rajaie Cardiovascular Medical and Research Center, Iran University of Medical Sciences, Tehran, Iran. Email: tatari@sbmu.ac.ir
}

Received 2018 September 07; Revised 2019 May 19; Accepted 2019 May 22.

\begin{abstract}
Introduction: Scimitar syndrome, a rare form of congenital heart disease (CHD), is characterized by anomalous pulmonary venous return into the inferior vena cava, hypoplasia of the right lung, and dextroposition of heart. The most common form of the syndrome known as infantile form, is diagnosed in infancy and is surgically corrected. Asymptomatic cases are occasionally diagnosed in adolescence. However, there are rare cases which remain undiagnosed until older ages despite some manifestations of the disease. This is in part due to the nonspecific nature of this manifestations which necessitates a specific workup for the diagnosis and treatment.

Case Presentation: We report two cases of scimitar syndrome in female patients, one 17 years old and another 48 years old. Here we described the symptoms, diagnosis and clinical course of these patients and reviewed the literature on Scimitar syndrome particularly in adolescents and adults.

Conclusions: Although Scimitar syndrome has early manifestations in most of the patients, its late diagnosis is sometimes seen in adolescents and adults due to misdiagnosis. Surgical repair can correct the blood flow route and improve the outcomes.

Keywords: Scimitar Syndrome, Heart Defects, Congenital, Scimitar Anomaly, Multiple Cardiac Malformations, Craniofacial and Central Nervous System Abnormalities [Supplementary Concept], Pulmonary Veins
\end{abstract}

\section{Introduction}

Congenital heart diseases (CHD) are accounted for congenital malformations in one percent of the live births and are the most prevalent type of the congenital disease (1). Major types of the CHD include tetralogy of Fallot, atrial septal defect, ventricular septal defect, and anomalies of the aorta and other great vessels of the heart $(1,2)$. Most of the severe cases with clinical manifestations are classified in the category of the cyanotic forms and diagnosed in early infancy and childhood. Surgical correction is performed to correct these anomalies $(1,3)$.

Scimitar syndrome is a congenital heart disease involving the pulmonary venous connection to the right heart in association with pulmonary abnormalities. Symptoms include hypoplasia of the right lung and pulmonary branch along with the sequestration of the right lower lobe as well as the large aortic collaterals $(4,5)$. The infantile form usually requires surgical intervention; however, the adult form is usually asymptomatic and surgical repair is dependent on the severity of the left-right shunt $(4,6,7)$. Timely diagnosis helps with the appropriate management of the patients, thus preventing the development of the severe forms of the adverse outcomes $(6,8)$. We present two cases of adult patients incidentally diagnosed with scimitar syndrome. These adults had been suspected and received treatment for complications like respiratory problems and the diagnosis of Scimitar sydrome was postponed until adolescence or adulthood.

\section{Case Presentation}

\subsection{Presentation of Case 1}

The first case was a 49-year-old female. The patient was diagnosed with pulmonary problems around 14 years ago. She also had endoscopy examination two years ago.

She underwent the workup for diagnosis of the cause of the pulmonary problem. The patient had dyspnea on exertion (DOE) with class II functional symptoms according to New York Heart Association (NYHA) functional classification. Other complications included hypertension and diabetes mellitus. The patient was taking the following 
medicines: Lantus, Zipmet (sitagliptin/metformin), atorvastatin, metoral, losartan, gliclazide, aspirin, peptazol, domperidone and vitamin B1. A diastolic S1-S2 murmur was heard on auscultation.

Transesophageal echocardiography demonstrated a normal sized left ventricle without hypertrophy and a mildly reduced systolic function. Left atrium was normal in echocardiography and right atrium was enlarged. Right ventricle was moderately enlarged and demonstrated a slightly reduced systolic function. Mitral valve appeared normal and without stenosis, however a mild mitral regurgitation was observed. Aortic valve was normal and without stenosis or aortic insufficiency. Mean pulmonary arterial pressure was $30 \mathrm{mmHg}$. Multislice computed tomography (MSCT) showed scimitar vein. Surgical repair was recommended to correct the left-to-right shunt, as a result of pulmonary venous drainage to the inferior vena cava.

Reparation of the Scimitar vein was performed according to the regular method for correction of Scimitar syndrome and anomalous vein was diverted from IVC to the left atrium through ASD. The patient was examined using echocardiography after operation. Correct routing of the anomalous vein to the left atrium without stenosis was observed.

\subsection{Presentation of Case 2}

The second case was a 16-year-old female. She had complaints about DOE. Patient underwent the workup for the diagnosis of the cause of the pulmonary problem. DOE was of functional class II according to the NYHA functional classification. Her blood pressure was 110/80 and pulse rate was $85 /$ minutes. She was presented with systolic murmur on auscultation. The patient had no other apparent presentation of the condition.

The patient underwent angiography with the following significant observations: $\mathrm{O}_{2}$ saturation percent in the aorta, pulmonary artery (PA) and the superior vena cava (SVC) were 96, 85, and $78 \mathrm{mmHg}$, respectively. This amount was $87 \%$ for inferior vena cava (IVC) which suggests a left to right shunt from pulmonary vein to IVC (Figure 1). Other significant angiographic observations included Scimitar vein of right heart catheter and secundum type atrial septal defect (ASD) as observed by the left side catheter. The LV size and diastolic function was normal. However, a mild mitral regurgitation was observed. Mean pulmonary arterial pressure of this patient was $40 \mathrm{mmHg}$.

Transesophageal echocardiography demonstrated a normal sized left ventricle without hypertrophy. Left atrium was normal in echocardiography and right atrium was enlarged. Right ventricle was severely enlarged and with mild systolic dysfunction. Mild enlargement of the right atrium was observed. Mitral valve appeared normal

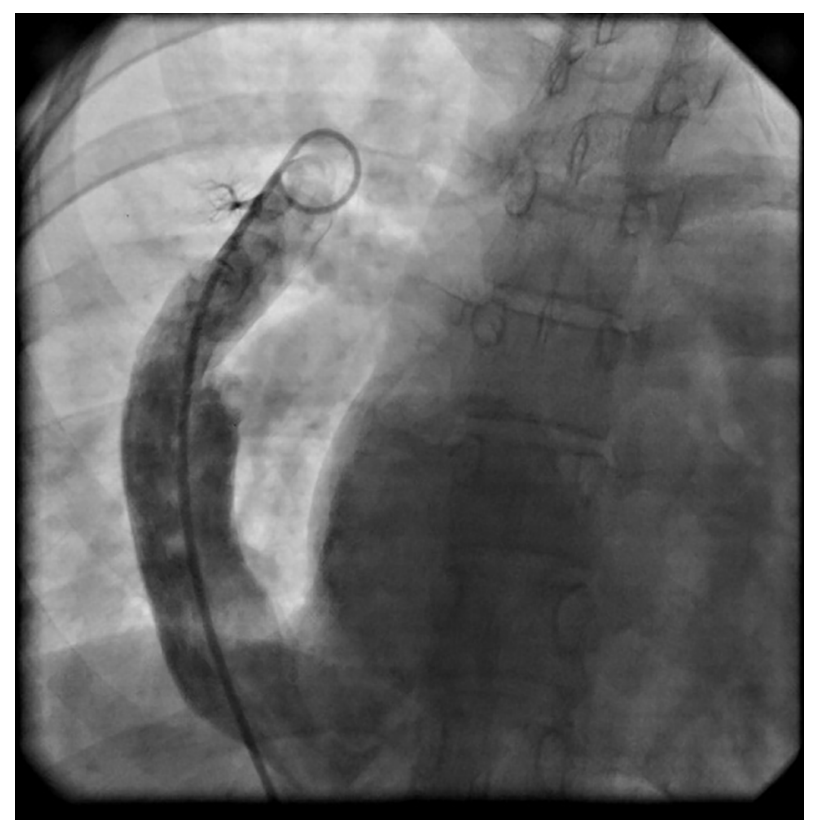

Figure 1. Scimitar vein draining into inferior vena cava (IVC)

and without stenosis with mild regurgitation. Aortic valve was normal and without stenosis or aortic insufficiency. Large secundum type ASD $(1.7 \mathrm{~cm})$ with a significant left to right shunt (QP/QS: 2.5) was observed. Right pulmonary vein was entered near the IVC entrance to the RA. Observations was consistent with the diagnosis of a large ASD and a Scimitar vein.

Multislice computed tomography showed scimitar vein and levocardia. Right (RAE) and left atrial enlargement (LAE), and central pulmonary artery dilatation were observed. Scimitar syndrome was noted as almost complete drainage of the right lung venous flow to the supra hepatic IVC. Only a small right sided subsegmental venous branch connects directly to the LA. All left pulmonary vein is drained into the LA.

The recommended treatment was surgical repair to correct the left-to-right shunt, as a result of pulmonary venous drainage to the inferior vena cava, and ASD closure. For this patient, reparation was also performed according to the routine approach for correction of Scimitar syndrome and anomalous vein was diverted from IVC to the left atrium through ASD. Echocardiographic examination of the patient after operation, demonstrated correct routing of the anomalous vein to the left atrium without stenosis. 


\section{Discussion}

Although scimitar syndrome is becoming more widely recognized, physicians may be unfamiliar with its variable presentation (8). Despite development of the surgical techniques for the management of the diseases, mortality remains high especially in the patients with other coexisting congenital malformations (9). Since late diagnosis hinders the timely intervention, patients may develop further acquired malformations and even heart failure in severe cases. Considering the serious risk of the associated morbidity and mortality, high index of suspicion for this condition must be maintained for timely diagnosis $(4,8)$. In the first case presented here patient was misdiagnosed and appropriate treatment was postponed for a long time. Beyond impairing the patients' quality of life, the disease can result in life threatening heart failure in long term. The syndrome should be suspected in cases with right-sided "atelectasis". Dextroposition of the heart and right lung hypoplasia are other conditions where scimitar syndrome is suspected with, although some cases of the disease in patients with levocardia are reported. Second case presented in this study was with normal heart positioning despite suffering from scimitar syndrome. This condition may further complicate the diagnosis of the syndrome.

Imaging modalities for diagnosis of the condition might include chest X-rays, percutaneous examination of the heart chambers, echocardiographic examination, and MSCT reconstruction and magnetic resonance imaging (MRI) of the chest (10).

On chest X-ray, heart is shifted into the right chest in a small fraction of adult patients and right hypoplastic lung is evident in most of the patients. "Scimitar sign"-a curvilinear opacity widening in its route to IVC, is presented on chest X-ray.

In case of these two patients Scimitar vein was evident in the reconstructed view of the MSCT and provided definitive diagnosis of the vein positioning.

According to the literature left-to-right shunt is the main indication for surgical intervention. This abnormality is present in more than $50 \%$ of the patients with Scimitar syndrome (10). Cases presented in the current study both were suffering from this abnormality, hence they needed surgical intervention for correction of the left-toright shunt. Co-abnormalities such as ASD may also be observed in this syndrome which is corrected with a pericardial patch through surgical operation (11). The second case presented here had ASD co-abnormality. This abnormality is more often observed in infantile form than adult form.

\subsection{Conclusions}

The routine workup for patients suspect with scimitar syndrome include history taking, physical examination, echocardiography, chest CT, or MRI and cardiac catheterization to diagnose the disease. Furthermore, understanding the pulmonary hemodynamics and other accompanying complications is of vital importance. After diagnosis, surgical correction of the malformations as the main treatment option should be prompted to prevent the onset of irreversible pulmonary hypertension.

\section{Footnotes}

Authors' Contribution: Study concept and design: Gholamreza Omrani, Maziar Gholampour Dehaki, Reza Abbaszadeh, Ali Sadeghpour Tabaee, and Hassan Tatari. Analysis and interpretation of data: Gholamreza Omrani, Maziar Gholampour Dehaki, Reza Abbaszadeh, Ali Sadeghpour Tabaee, and Hassan Tatari. Drafting of the manuscript: Hassan Tatari. Critical revision of the manuscript for important intellectual content: Gholamreza Omrani, Maziar Gholampour Dehaki, Reza Abbaszadeh, and Ali Sadeghpour Tabaee.

Conflict of Interests: The authors have no conflict of interest.

Funding/Support: No funding to declare.

Patient Consent: Patients' informed consent was taken for publishing the data.

\section{References}

1. Triedman JK, Newburger JW. Trends in congenital heart disease: The next decade. Circulation. 2016;133(25):2716-33. doi: 10.1161/CIRCULATIONAHA.116.023544. [PubMed: 27324366].

2. Hoffman JI, Kaplan S, Liberthson RR. Prevalence of congenital heart disease. Am Heart J. 2004;147(3):425-39. doi: 10.1016/j.ahj.2003.05.003. [PubMed: 14999190].

3. Gorjipour F, Dehaki MG, Totonchi Z, Hajimiresmaiel SJ, Azarfarin $\mathrm{R}$, Pazoki-Toroudi $\mathrm{H}$, et al. Inflammatory cytokine response and cardiac troponin I changes in cardiopulmonary bypass using two cardioplegia solutions; del Nido and modified St. Thomas': A randomized controlled trial. Perfusion. 2017;32(5):394-402. doi: 10.1177/0267659117691119. [PubMed: 28152655].

4. Cicek S, Arslan AH, Ugurlucan M, Yildiz Y, Ay S. Scimitar syndrome: The curved Turkish sabre. Semin Thorac Cardiovasc Surg Pediatr Card Surg Annu. 2014;17(1):56-61. doi: 10.1053/j.pcsu.2014.01.003. [PubMed: 24725718].

5. Lugones I, Biancolini MF, Zerpa Pacheco VE, Martinez IA, Damsky Barbosa JMM, de Dios AMS. Modified in situ pericardial rerouting technique for scimitar syndrome repair. World J Pediatr Congenit Heart Surg. 2017;8(6):735-9. doi: 10.1177/2150135117732540. [PubMed: 29187109].

6. [No authors listed]. Corrigendum to: The natural history and surgical outcome of patients with scimitar syndrome: A multi-centre European study. Eur Heart J. 2019;40(2):179. doi: 10.1093/eurheartj/ehy298. [PubMed: 29800132]. 
7. Masrani A, McWilliams S, Bhalla S, Woodard PK. Anatomical associations and radiological characteristics of Scimitar syndrome on CT and MR. J Cardiovasc Comput Tomogr. 2018;12(4):286-9. doi: 10.1016/j.jcct.2018.02.001. [PubMed: 29550261].

8. Mordue BC. A case series of five infants with scimitar syndrome. Adv Neonatal Care. 2003;3(3):121-32. [PubMed: 12891836].

9. Gupta ML, Bagarhatta R, Sinha J. Scimitar syndrome: A rare disease with unusual presentation. Lung India. 2009;26(1):26-9. doi: 10.4103/0970-2113.45202. [PubMed: 20165592]. [PubMed Central:
PMC2813113].

10. Huang SF, Yu WC, Chern JH, Lee YC. Scimitar syndrome in an older adult. J Chin Med Assoc. 2011;74(11):516-9. doi: 10.1016/j.jcma.2011.09.008. [PubMed: 22100022].

11. Rajaii-Khorasani A, Kahrom M, Mottaghi H, Kahrom H. Scimitar syndrome: Report of a case and its surgical management. Ann Saudi Med. 2009;29(1):50-2. doi: 10.4103/0256-4947.51820. [PubMed: 19139615]. [PubMed Central: PMC2813621]. 